\title{
N89-15607
}

\section{SPACECRAFT ENVIRONMENTAL ANOMALIES EXPERT SYSTEM}

\author{
H. C. Koons and D. J. Gorney \\ Space Sciences Laboratory \\ The Aerospace Corporation \\ Mail Station M2-260 \\ P.O Box 92957 \\ Los Angeles, Ca. 90009
}

\begin{abstract}
A micro-computer-based expert system is being developed at the Aerospace Corporation Space Sciences Laboratory to assist in the diagnosis of satellite anomalies caused by the space environment. The expert system is designed to address anomalies caused by surface charging, bulk charging, single event effects and total radiation dose. These effects depend on the orbit of the satellite, the local environment (which is highly variable), the satellite exposure time and the "hardness" of the circuits and components of the satellite. The expert system is a rule-based system that uses the Texas Instruments Personal Consultant Plus expert system shell. The completed expert system knowledge base will include 150-200 rules, as well as a spacecraft attributes database, an historical spacecraft anomalies database, and a space environment database which is updated in near-real-time. Currently, the expert system is undergoing development and testing within the Aerospace Corporation Space Sciences Laboratory.

\section{INTRODUCTION}

The Aerospace Corporation Space Sciences Laboratory (SSL) is currently involved in the development and testing of a micro-computer-based expert system to aid in the objective analysis of environmentally-induced satellite anomalies. On orbit anomalies in satellite systems or subsystems occur quite often (several hundred are reported each year), and the number, frequency and severity of the anomalies are likely to grow with the inevitable increases in spacecraft complexity in the future. Spacecraft anomalies have a wide variety of causes and wide ranges of effects and severity. Design errors and inadequate quality control in parts selection and workmanship are examples of pre-flight engineering and construction errors which can lead to later anomalous behavior of components on orbit. Naturally, all mechanical and electrical components are susceptible to failure from wearout. Wearout failures and their effects can be difficult or impossible to anticipate. Many satellite anomalies result directly from improper commanding or operation (human error or ground system error). For Defense Department space systems, the possibility of hostile action must be a consideration as well. Also, recent studies have shown that adverse interactions between spacecraft components and the natural space environment can have deleterious consequences comparable in severity and frequency to those caused by any other factor. Indeed, spacecraft anomalies attributable to electrostatic discharges (just one form of environmental interaction) have been known to cause command errors, spurious signals, phantom commands, degraded sensor performance, part failure and even complete mission loss. Regardless of the severity of the anomaly, it is important to assess the cause of the problem in a timely and accurate manner so that appropriate corrective action can be taken.

Various aspects of the space environment can cause on-orbit satellite anomalies. The plasma environment (especially in geosynchronous orbit) can cause differential charging of satellite components on the surface of the vehicle. Surface charging can exceed breakdown voltages, and electrostatic discharges (ESDs) can occur with the potential to disrupt electronic components. More energetic components of the space radiation environment can penetrate and become embedded in
\end{abstract}




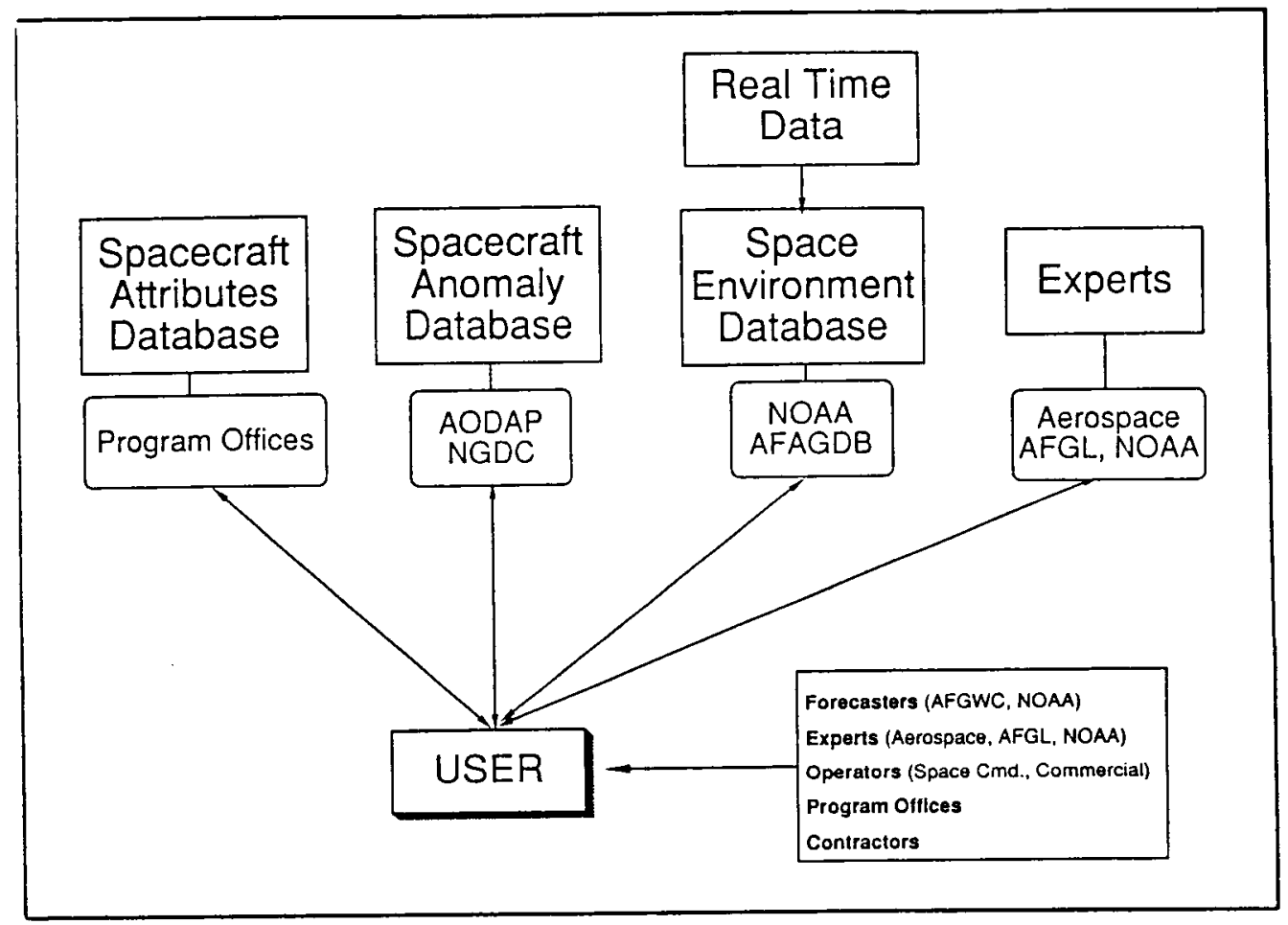

Figure 1a

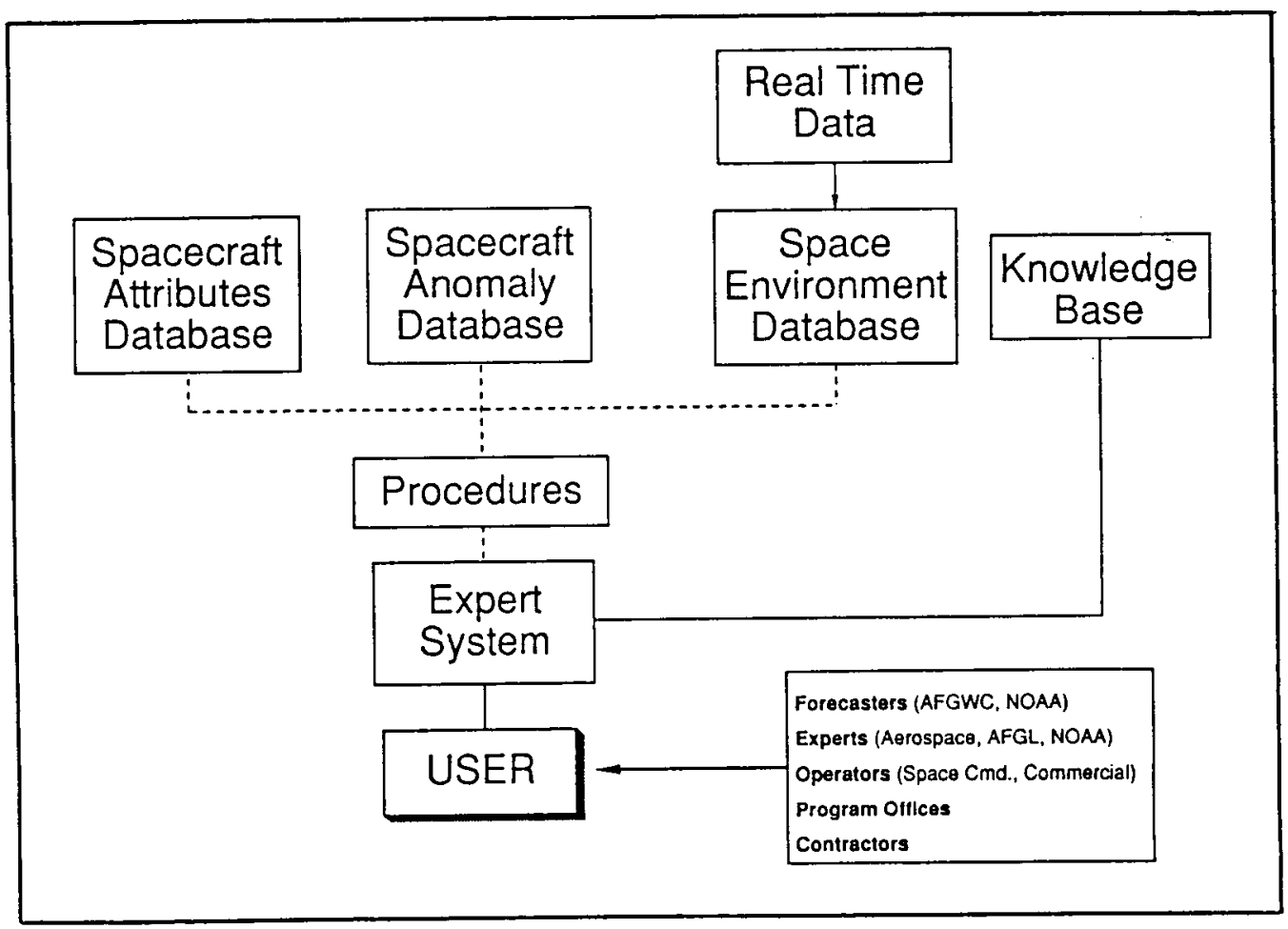

Figure 1b 
dielectric components such as cable insulation and circuit boards. This "bulk charging" phenomenon can, under extreme circumstances, result in ESD within the dielectric components, disrupting signals or devices within the affected subsystem. Trapped radiation belt particles, solar flare protons and galactic cosmic rays can cause single event upsets (SEU) within microelectronic devices. This same high energy radiation leads to degradation of microelectronic devices and sensors through total dose effects. Other aspects of the environment, such as micrometeors and debris, can cause mechanical disruption of the vehicle. Anomalies which result from any of these environmental causes can lead to transient malfunction or even to non-recoverable loss of the component or subsystem.

Typically, various agencies and individuals become involved in the identification and resolution of spacecraft anomalies. These include (1) the commercial or military satellite operators who must evaluate the anomaly in near real time in order to take proper corrective action or to "safe" the vehicle; (2) space-environment forecasters, such as the Air Force Global Weather Central (AFGWC) or the NOAA Space Environment Services Center (SESC), who must assess the environmental situation in real time and issue warnings and alerts regarding hazardous conditions; (3) satellite contractors, who must assess the susceptibility of their vehicle and incorporate design modifications if the vehicle's on-orbit reliability proves to be inadequate; and (4) scientists and engineers who develop an understanding of the processes by which the environment interacts with the satellite, with the goal of recommending mitigating procedures for future missions. A major difficulty with the spacecraft anomaly diagnosis problem is that many of the operational groups who must quickly and accurately diagnose the anomaly do not have immediate access to the required data or to the scientific or engineering expertise required to properly assess the role of the space environment in the anomaly. Thus, real-time spacecraft anomaly diagnosis appears to be an ideal application for an expert system which can gather, format, display and utilize appropriate data consistent with logical rules based on state-of-the-art engineering and scientific expertise. The Aerospace Corporation Space Sciences Laboratory, based on its long-term involvement in space environment research and its continuous interactions with the satellite operational community, has undertaken the task of developing such an expert system.

\section{SYSTEM DESCRIPTION}

The Spacecraft Environmental Anomalies Expert System has been developed within the framework of the Texas Instrument's Personal Consultant Plus expert system shell, and has been implemented for development and test on a Compac Deskpro 386 computer. The basic architecture of the system is designed to conform as much as possible to the working environment in which the system will ultimately be implemented while taking advantage of the data-processing, data display and decision-making functions of the computer system. The design also makes use of existing data sets. Figure 1a shows the typical user environment without the benefit of an expert system. A user (anyone who is responsible for the diagnosis of a satellite anomaly) generally has access to a number of data bases and to some amount of expertise. The data bases might be computer-based or in the form of technical literature; the data bases might be on-site or at other agencies. The most pertinent data bases are the Space Environment Data Base, which contains historical and real-time data on the space environment; the Spacecraft Attributes Data Base, which contains information on the vehicles (this might include component information, ephemerides, etc.); and a Spacecraft Anomaly Data Base containing records of previous anomalies on the vehicle in question. Expert opinions are usually available over the phone or in consultations after the fact. Even under the best of circumstances the user has a formidable task to acquire and digest the information pertinent to his diagnosis. Often the user may not know what information is available or what information is pertinent.

Figure 1b shows the implementation of the Spacecraft Environmental Anomaly Expert System. The expert system not only provides access to a consolidated interactive knowledge base, but also provides procedures to access and display information from the data bases. The knowledge base, currently consisting of over 100 logical rules, has been constructed based on personal interviews with space scientists and engineers. In practice, the expert system could be operated (albeit will diminished effectiveness) even if one or more of the prescribed data bases were un- 
available. At present, the expert system is configured to diagnose anomalies caused by surface charging, bulk charging, single event effects and total radiation dose. The system could be expanded to include a broader range of environmental causes of anomalies without any difficulty. The performance of the system has been tested on historical case studies, and the following section gives a typical example of a single consultation with the expert system.

\section{SAMPLE CONSULTATION}

We will follow a typical consultation with the Spacecraft Environmental Anomalies Expert System by showing a selection of representative screens through which the user interacts with the system. First the user selects the Expert or Novice mode. The Novice mode contains more descriptive material and antecedent rules that explain the conclusions reached by the system. It is intended as a learning tool.

The user next selects from Fig. 2 the data bases that he wishes to use during the consultation. This also permits a flexible configuration at different sites where one or more of the databases may not be available. The four databases shown in Fig. 2 represent different techniques for storing and accessing data. The Anomaly database is a Dbase III Plus file provided by the Na-

Select all of the databases that are available for this system.

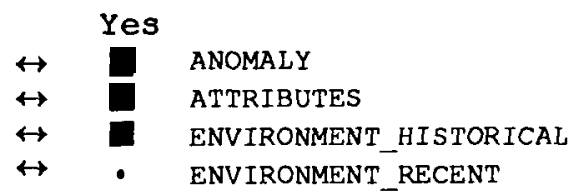

1. Use arrow keys or first letter of item to position cursor.

2. Select all applicable responses.

3. After making selections, press RETURN/ENTER to continue.

Figure 2

The maximum value for $\mathrm{Kp}$ during the four day period ending with the date of the anomaly was 8.3

The convention used is the following: $\mathrm{Kp}$ of $3^{-}=2.7, \mathrm{Kp}$ of 3 is 3.0 , and $\mathrm{kp}$ of $3+$ is 3.3 .

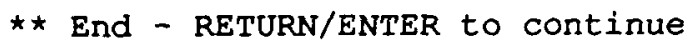


The name of the satellite in the anomaly database mey be different from the name in the other databases. Please select the name of the satellite for the anomaly report from this list. If the name does not appear in this list then the satellite is not in the anomaly database. In that case choose UNKNOWN.

$\begin{array}{lllll}\text { @GG0402 } & \text { @GG0401 } & \text { GOES-5 } & \text { @GG0508 } & \text { AUSSAT-A3 } \\ \text { @GG0403 } & \text { METEOSAT-1 } & \text { METEOSAT-2 } & \text { INSAT-1B } & \text { SCATHA } \\ \text { @GG0404 } & \text { @GG0414 } & \text { GMS-2 } & \text { NAVSTAR } & \text { UNKNOWN } \\ \text { @GG0407 } & \text { @PN0103 } & \text { @GG0503 } & \text { GOES-6 } & \\ \text { DSCS-9434 } & \text { @GG0416 } & \text { MARECS-A } & \text { GMS-3 } & \\ \text { DSCS-9433 } & \text { GMS } & \text { @GG0504 } & \text { @PN0302 } & \\ \text { @PN0102 } & \text { @PN0101 } & \text { @GG0505 } & \text { @PN0402 } & \\ \text { @GW0101 } & \text { @PN0201 } & \text { TDRSS-1 } & \text { AUSSAT-A1 } & \\ \text { @GG0408 } & \text { @GG0413 } & \text { @GG0506 } & \text { AUSSAT-A2 } & \\ \text { @GG0411 } & \text { EGG0502 } & \text { @PN0303 } & \text { KU2 } & \\ \text { @GG0412 } & \text { GGG0501 } & \text { @PN0401 } & \text { GOES-7 } & \end{array}$

1. Use the arrow keys or first letter of item to position cursor.

2. Press RETURN/ENTER to continue.

\section{Figure 4}

tional Geophysical Data Center in Boulder, CO. It presently contains a record of approximately 2000 historical anomalies. The Attributes database consists of a text (ASCII) file for each of a small selection of satellites. It contains orbital information. The Environment_Historical database is a binary file that contains an historical record of the geophysical parameter known as $\mathrm{Kp}$, the planetary magnetic index. $\mathrm{Kp}$ is a measure of the severity of magnetic storms within the Earth's magnetosphere. This file is accessed by a C-language interface between the expert system and the binary file. An example of the output from the file is shown in Fig. 3. Kp is measured on a scale from 0 to 9. The large value, 8.3, shown in Fig. 3 means that a severe storm occurred during the time period.

The Environment_Recent database has not yet been implemented. It is planned to be a binary file similar to the Environment_Historical file. The recent data will be collected by a remote computer from the satellite broadcasts by the Space Environment Services Center, Boulder, CO. When needed the most recent data in the file will be automatically transferred via telephone modem to the consultation computer.

If the user chooses to use the Anomaly database the expert system automatically queries the database via a Dbase III interface and obtains a list of the satellites that are contained in the database. It then presents the satellite selection screen shown in Fig. 4 to the user. Many of the names are coded to conceal the identity of the actual vehicle. Two types of reports are generated. A satellite report lists all of the anomalies in the database for a single vehicle. This can be used to search for recurrences of similar anomalies. The recurrence in specific local time sectors or in limited regions of the orbit for example is an indication of a possible environmental cause. A data report such as the one shown in Fig. 5 lists of all of the anomalies in the database for a three day time period around the date entered by the user. Frequently more than one vehicle is affected by a severe storm. The occurrence of similar anomalies on more than one vehicle in a short time period is an indication of a possible environmental cause.

The expert system provides the user direct access to the Spacecraft Anomaly Manager software (SAM) which has been developed by the National Geophysical Data Center. The SAM utility provides a full range of functions for managing, displaying and analyzing the data, including functions to test single anomalies or sets of anomalies for environmental relationships. Histograms of local time and seasonal occurrence frequency provided by this utility can reveal distinct patterns 


\begin{tabular}{|c|c|c|c|c|c|}
\hline \multicolumn{2}{|l|}{ Page No. 1} & \multicolumn{2}{|c|}{ SPACECRAFT ANOMALY } & \multicolumn{2}{|c|}{ REPORT } \\
\hline SATELLITE & DATE & LT & TYPE & DIAG & COMMENT \\
\hline METEOSAT-2 & $09 / 22 / 82$ & 0522 & PC & ESD & Two parameters \\
\hline eGG0504 & $09 / 22 / 82$ & 1422 & sS & ESD & 12 GHz TWTA shutdown \\
\hline EGG0407 & $09 / 23 / 82$ & 0429 & SE & SEU & \\
\hline eGG0407 & $09 / 23 / 82$ & 0519 & SE & SEU & \\
\hline egG0407 & $09 / 23 / 82$ & 0611 & SE & SEU & \\
\hline METEOSAT-2 & $09 / 23 / 82$ & 0739 & PC & ESD & Three parameters \\
\hline GOES-4 & $09 / 23 / 82$ & 0106 & PC & ESD & VISSR STEP SCAN ON \\
\hline
\end{tabular}

\section{Figure 5}

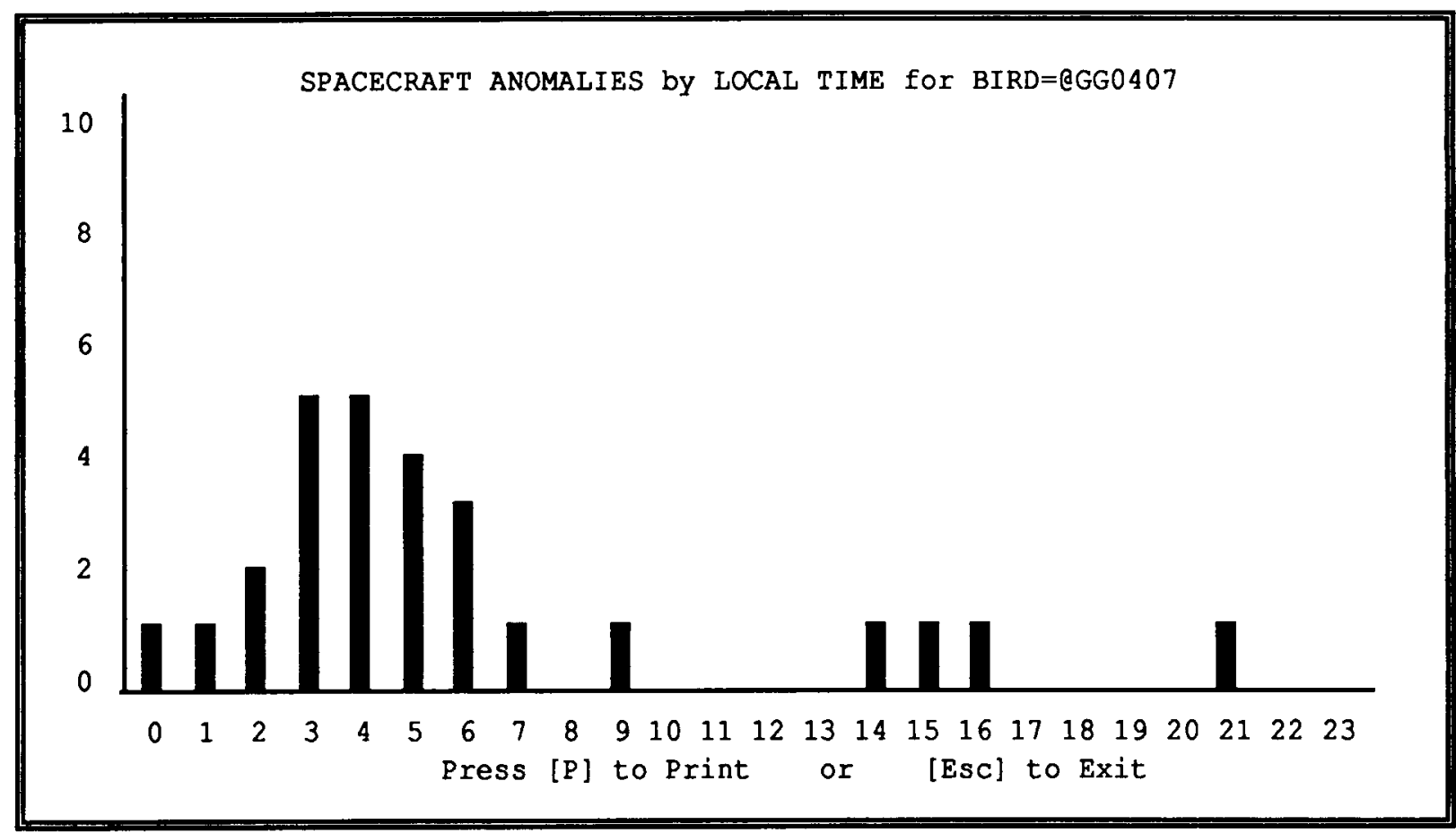

Figure 6

for spacecraft which are susceptible to static charge buildup and ESD. Figure 6 shows one example of the graphical output available from SAM. The SAM utility also provides a means of updating the Anomaly Database after the expert system consultation.

As progress is made through the consultation a number of specific questions are asked. Fig. 7 shows the screen for selecting one or more types of problems associated with the anomaly under study. The system presently has four major frames for each of the four major environmental causes of anomalies, surface charging, bulk charging, single event upset and total radiation dose. The responses to this screen are used to instantiate the frames.

Fig. 8 shows a typical question regarding the occurrence of a large solar x-ray flare. The option to answer "unknown" is always offered to the user. This may elicit another question on the same topic or cause the system to abandon that line of chaining. 
Select all of the types of problems that are associated with this anomaly.

$\begin{array}{lcll} & \text { Yes } & \\ \leftrightarrow & \bullet & \text { PHANTON_COMMAND } \\ \leftrightarrow & \cdot & \text { LOGIC_UPSET } \\ \leftrightarrow & \cdot & \text { ELECTRICAL } \\ \leftrightarrow & \cdot & \text { MECHANICAL } \\ \leftrightarrow & \square & \text { SENSOR } \\ \leftrightarrow & \cdot & \text { SOFTWARE } \\ \leftrightarrow & : & \text { MEMORY } \\ \leftrightarrow & \cdot & \text { THERMAL } \\ \leftrightarrow & \cdot & \text { PART_FAILURE } \\ \leftrightarrow & \cdot & \text { TELEMETRY_ERROR } \\ \leftrightarrow & \cdot & \text { SYSTEM_FAILURE } \\ \leftrightarrow & \cdot & \text { MISSION_FAILURE } \\ \leftrightarrow & \cdot & \text { OTHER }\end{array}$

1. Use arrow keys or first letter of item to position cursor.

2. Select all applicable responses.

3. After making selections, press RETURN/ENTER to continue.

Figure 7

Did a very large solar $x$-ray flare e.g. a type $X 1$ solar $x$-ray flare occur within a few hours prior to the anomaly?

YES

NO

UNKNOWN

1. Use the arrow keys or first letter of item to position the cursor.

2. Press RETURN/ENTER to continue.

Figure 8

Figure 9 shows a highly technical question regarding the level for the accumulation of energetic electrons in the vehicle. This requires access to satellite environmental data plus an analysis or expert opinion to relate the measurements from the satellite making the measurements to the one experiencing the anomaly. Help is always available by pressing function key F1. The help window then appears as shown in Fig. 9. The help window contains a more detailed explanation of the question and a person or organization to contact for assistance.

Single event upsets are caused by the deposition of energy in digital devices when a very energetic particle passes through the device. The probability of an anomaly thus depends on the hardness of the device as well as on the environment. Fig. 10 shows a list of devices that is displayed for the user if the Single Event Upset frame is instantiated. A series of rules in this frame contains qualitative information on the hardness of each type of technology as determined by laboratory measurements of their upset cross sections. 
Select the appropriate level for the accumulated fluence of energetic

electrons above $300 \mathrm{keV}$ for several days prior to the anomaly.

VERY_HIGH

HIGH

INTERMEDIATE

LOW

UNKNOWN
Help:

The accumulated fluence of penetrating electrons is the integral of the electron flux above 300 $\mathrm{keV}$ for several days before the anomaly. It is measured in units of [electrons/ $\mathrm{cm}^{\wedge} 2$ ]. For assistance in determining the fluence contact $D$. Gorney (213/336-6821) at the Aerospace Corp.

$$
\begin{aligned}
& \text { VERY_HIGH }>10^{\wedge} 12 \text { HIGH } 10^{\wedge} 11-10^{\wedge} 12 \\
& \text { INTERMEDIATE } 10 \wedge 10-10 \wedge 11 \text { LOW <10^10 } \\
& \text { ** End - RETURN/ENTER to continue }
\end{aligned}
$$

1. Use the arrow keys or first letter of item to position the cursor.

2. Press RETURN/ENTER to continue.

\section{Figure 9}

Select the technology which best describes the softest devices in the circuit experiencing the anomaly.

$\begin{array}{ll}\text { ADV_CMOS } & \text { IIL } \\ \text { ADV_HCMOS } & \text { TTL } \\ \text { ADV_SCHOTTKY } & \text { ALS/TTL } \\ \text { CMOS } & \text { ECL/TTL } \\ \text { CMOS/EPI } & \text { L/TTL } \\ \text { CMOS/SOS } & \text { LS/TTL } \\ \text { HCMOS } & \text { S/TTL } \\ \text { LOC/MOS } & \text { SCHOTTKY } \\ \text { MOS } & \text { OTHER } \\ \text { NMOS } & \\ \text { NMOS/CMOS } & \\ \text { NMOS/EPI } & \\ \text { NMOS/SOS } & \\ \text { PMOS } & \end{array}$

1. Use the arrow keys or first letter of item to position cursor.

2. Press RETURN/ENTER to continue.

Figure 10

Finally Fig. 11 shows the conclusion screen. A confidence level is given for each possible cause. Note that a negative conclusion is listed whenever a specific cause can be ruled out.

The user may then review the consultation with the option to modify one or more of his responses to see how they affect the conclusion. Also, the user may choose to re-enter the Spacecraft Anomaly Manager utility to update the Anomaly Database based on the results of the consultation. 


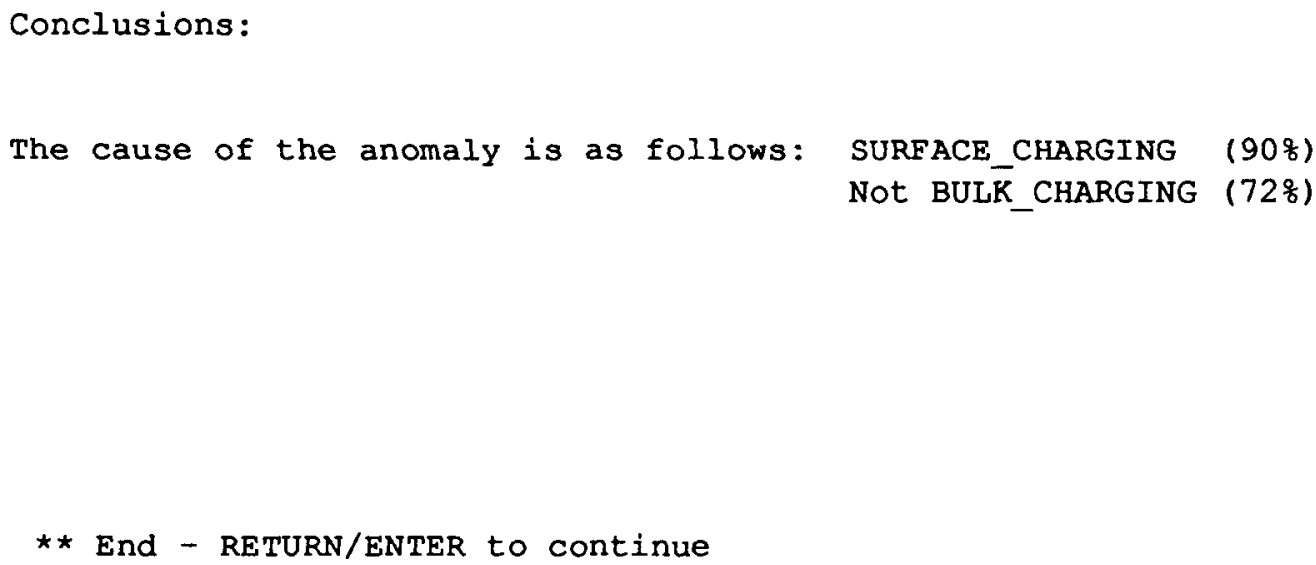

Figure 11

\section{SUMMARY AND STATUS}

The Spacecraft Environmental Anomalies Expert System is currently undergoing development and testing at the Aerospace Corporation Space Sciences Laboratory. The system aids in the analysis of satellite anomalies which may be caused by interactions with the space environment. Currently, the system deals with anomalies caused by electrostatic discharges resulting from surface or bulk dielectric charging, single event upsets, and total radiation dose. A prototype system has been developed, including three databases and a knowledge base consisting of about one hundred rules. The expert system also contains software to access, display and modify the data. The completed system will consist of approximately $150-200$ rules. We expect that the expert system will be available for implementation at operational sites sometime during 1989. Potential users of the system include space environment forecasters at the Air Force Global Weather Central, civilian and military satellite operators, and spacecraft contractors.

\section{ACKNOWLEDGEMENTS}

We benefitted greatly from technical discussions with Drs. Joe H. Allen, Gary Heckman and Dan Wilkinson at NOAA and with Drs. J. Fennell, A. Vampola, and W. A. Kolasinski at the Aerospace Corporation. The Spacecraft Anomaly Manager software used in our expert system is provided by the NOAA National Geophysical Data Center. This project was supported by the Aerospace Sponsored Research Program and by the US Air Force System Command's Space Division under Contract No. F04701-86-C-0087 through the Satellite Control Network Program Office. 